\title{
ARTICLE OPEN \\ Observation of non-locality sharing among three observers with one entangled pair via optimal weak measurement
}

\author{
Meng-Jun $\mathrm{Hu}^{1,2}$, Zhi-Yuan Zhou $\mathbb{D}^{1,2}$, Xiao-Min Hu${ }^{1,2}$, Chuan-Feng $\mathrm{Li}^{1,2}$, Guang-Can Guo ${ }^{1,2}$ and Yong-Sheng Zhang $\mathbb{D}^{1,2}$
}

Bell non-locality plays a fundamental role in quantum theory. Numerous tests of the Bell inequality have been reported as the ground-breaking discovery of the Bell theorem. Up to now, however, most discussions of the Bell scenario have focused on a single pair of entangled particles distributed to only two separated observers. Recently, it has been shown surprisingly that multiple observers can share the non-locality from an entangled pair using the method of weak measurement without post-selection [Phys. Rev. Lett. 114, 250401 (2015)]. Here we report an observation of double CHSH-Bell inequality violations for a single pair of entangled photons with strength continuous-tunable optimal weak measurement in a photonic system. Our results shed new light on the interplay between non-locality and quantum measurements and our design of weak measurement protocol may also be significant for important applications such as unbounded randomness certification and quantum steering.

npj Quantum Information (2018)4:63; https://doi.org/10.1038/s41534-018-0115-x

\section{INTRODUCTION}

Non-locality, which was pointed out by Einstein, Podolsky and Rosen (EPR), ${ }^{1}$ plays a fundamental role in quantum theory. It has been intensively investigated as the ground-breaking discovery of Bell theorem by John Bell in 1964. ${ }^{2}$ Bell theorem states that any local-realistic theory cannot reproduce all the predictions of quantum theory and gives an experimental testable inequality ${ }^{3}$ that later improved by Clauser, Horne, Shimony and Holt (CHSH). ${ }^{4}$ Numerous tests of $\mathrm{CHSH}$-Bell inequality have been realized in various quantum systems $s^{5-13}$ and strong loophole-free Bell tests have been reported recently. ${ }^{14-16}$

To date, however, most discussions of Bell scenario focus on one pair of entangled particles distributed to only two separated observers Alice and Bob. ${ }^{17}$ It is thus an important and fundamental question whether or not multiple observers can share the non-locality from an entangled pair. Using the concept of weak measurement without post-selection, Silva et al. give a surprising-positive answer to above question and show a marvellous physical fact that measurement disturbance and information gain of a single system are closely related to nonlocality distribution among multiple observers in one entangled pair. $^{18}$

In this article, we report an experimental realization of sharing non-locality among three observers with strength continuoustunable optimal weak measurement in a photonic system. We produce pairs of polarization-entangled photons in our experiment and send it to Alice and Bob1, Bob2 separately, in this case two Bobs access the same single particle from the entangled pairs with Bob1 performs weak measurement. The realization of sharing non-locality is certified by the observed double violations of $\mathrm{CHSH}-$ Bell inequality among Alice-Bob1 and Alice-Bob2. The reason behind this is that weak measurement performed by Bob1 can be strong enough to obtain quantum correlations between Alice and Bob1, and weak enough to retain quantum correlations between Alice and Bob2. Our results not only shed new light on the interplay between non-locality and quantum measurements but also could find significant applications such as in unbounded randomness certification ${ }^{19,20}$ and quantum steering. $^{21,22}$

\section{RESULTS}

As one of the foundations of quantum theory, the measurement postulate states that upon measurement, a quantum system will collapse into one of its eigenstates, with the probability determined by the Born rule. Whereas this type of strong measurement, which is projective and irreversible, obtains the maximum information about a system, it also completely destroys the system after the measurement. Weak measurement, i.e., the coupling between the system and the probe is weak, however, can be used to extract less information about the system with less disturbance. It should be noted that this kind of weak disturbance measurement combined with post selection usually refers to weak measurement, ${ }^{23}$ which has been shown to be a powerful method in signal amplification, ${ }^{24-26}$ state tomography ${ }^{27,28}$ and in solving quantum paradoxes ${ }^{29}$ over the past decades. Hereafter, we follow the definition in ref. ${ }^{18}$ where weak measurement just refers to the measurement with intermediate coupling strength between the system and the probe. In contrast to strong projective measurement, weak measurement is non-destructive and retains some original properties of the measured system, e.g., coherence and entanglement. Because the entanglement is not completely destroyed by weak measurement, a particle that has been measured with intermediate strength can still be entangled with other particles, and therefore, sharing non-locality among multiple observers is possible.

\footnotetext{
${ }^{1}$ Laboratory of Quantum Information, University of Science and Technology of China, Hefei 230026, China and ${ }^{2}$ CAS Center for Excellence in Quantum Information and Quantum Physics, University of Science and Technology of China, Hefei 230026, China

Correspondence: Y-S. Zhang (yshzhang@ustc.edu.cn)

These authors contributed equally: Meng-Jun Hu, Zhi-Yuan Zhou.
}

Received: 5 August 2018 Accepted: 16 November 2018

Published online: 03 December 2018 

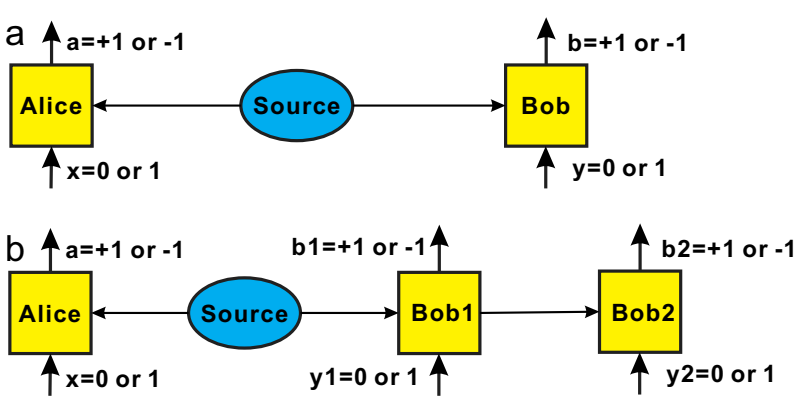

Fig. 1 Bell test. a Typical Bell scenario in which one pair of entangled particles is distributed to only two observers: Alice and Bob. b Modified Bell scenario in which Bob1 and Bob2 access the same single particle from the entangled pair with Bob1 performs a weak measurement

Consider a von Neumann-type measurement ${ }^{30}$ on a spin-1/2 particle that is in the superposition state $|\psi\rangle=a|\uparrow\rangle+\beta|\downarrow\rangle$ with $\mid$ $\left.a\right|^{2}+|\beta|^{2}=1$, where $|\uparrow\rangle(|\downarrow\rangle)$ denotes the spin up (down) state. After the measurement, the spin state is entangled with the pointer's state, i.e., $|\psi\rangle \otimes|\phi\rangle \rightarrow a|\uparrow\rangle \otimes\left|\phi_{\uparrow}\right\rangle+\beta|\downarrow\rangle \otimes\left|\phi_{\downarrow}\right\rangle$, where $|\phi\rangle$ is the initial state of the pointer and $\left|\phi_{\uparrow}\right\rangle\left(\mid \phi_{\downarrow}\right)$ indicates the measurement result of spin up (down). By tracing out the state of the pointer, the spin state becomes

$\rho=F \rho_{0}+(1-F)\left(\pi_{\uparrow} \rho_{0} \pi_{\uparrow}+\pi_{\downarrow} \rho_{0} \pi_{\downarrow}\right)$,

where $\rho_{0}=|\psi\rangle\left\langle\psi\left|, \pi_{\uparrow}=\right| \uparrow\right\rangle\left\langle\uparrow\left|, \pi_{\downarrow}=\right| \downarrow\right\rangle\langle\downarrow|$ and $F=\left\langle\phi_{\downarrow} \mid \phi_{\uparrow}\right\rangle$. The quantity $F$ is called the measurement quality factor because it measures the disturbance of the measurement. ${ }^{18}$ If $F=0$, the spin state is reduced to a completely decoherent state in the measurement eigenbasis, representing a strong measurement; otherwise, if $F=1$, there is no measurement at all. For other cases, i.e., $F \in(0,1)$, it refers to the measurement with intermediate strength called weak measurement.

Another important quantity associated with weak measurement is the information gain $G$ that is determined by the precision of the measurement. ${ }^{18}$ In the case of strong measurement, the probability of obtaining the outcome $+1(-1)$ that corresponds to spin eigenstate $|\uparrow\rangle(|\downarrow\rangle)$ can be calculated by the Born rule $P(+1)$ $=\operatorname{Tr}\left(\pi_{\uparrow} \rho_{0}\right)\left(P(-1)=\operatorname{Tr}\left(\pi_{\downarrow} \rho_{0}\right)\right)$. However, the non-orthogonality of the pointer states $\left\langle\phi_{\uparrow} \mid \phi_{\downarrow}\right\rangle \neq 0$ in weak measurement results in ambiguous outcomes. An observer who performs a weak measurement must choose a complete orthogonal set of pointer states $\left\{\left|\phi_{+1}\right\rangle,\left|\phi_{-1}\right\rangle\right\}$ as reading states to define the outcomes $\{+1$, $-1\}$ corresponding to the spin eigenstates $\{|\uparrow\rangle,|\downarrow\rangle\}$. The probabilities of the outcome \pm 1 in weak measurement then become $P( \pm 1)=\operatorname{Tr}\left(\pi_{\uparrow} \rho_{0}\right)\left|\left\langle\phi_{ \pm 1} \mid \phi_{\uparrow}\right\rangle\right|^{2}+\operatorname{Tr}\left(\pi_{\downarrow} \rho_{0}\right)\left|\left\langle\phi_{ \pm 1} \mid \phi_{\downarrow}\right\rangle\right|^{2}$. Here, | $\left.\left\langle\phi_{+1} \mid \phi_{\uparrow}\right\rangle\right|^{2}$ and $\left|\left\langle\phi_{-1} \mid \phi_{\downarrow}\right\rangle\right|^{2}$ correspond to the probabilities of obtaining the correct outcomes while $\left|\left\langle\phi_{-1} \mid \phi_{\uparrow}\right\rangle\right|^{2}$ and $\left|\left\langle\phi_{+1} \mid \phi_{\downarrow}\right\rangle\right|^{2}$ correspond to the probabilities of the wrong outcomes. For simplicity, we consider the case of symmetric ambiguousness in which $\left|\left\langle\phi_{+1} \mid \phi_{\uparrow}\right\rangle\right|^{2}=\left|\left\langle\phi_{-1} \mid \phi_{\downarrow}\right\rangle\right|^{2}$ and $\left|\left\langle\phi_{-1} \mid \phi_{\uparrow}\right\rangle\right|^{2}=\left|\left\langle\phi_{+1} \mid \phi_{\downarrow}\right\rangle\right|^{2}$, thus, the probabilities of the outcomes can be reformulated as

$P( \pm 1)=G \cdot \frac{1}{2}\left[1 \pm \operatorname{Tr}\left(\sigma \rho_{0}\right)\right]+(1-G) \cdot \frac{1}{2}$,

where $\sigma=\pi_{\uparrow}-\pi_{\downarrow}$ defines the spin observable and $G=1-\mid\left\langle\phi_{-1}\right|$ $\left.\phi_{\uparrow}\right\rangle\left.\right|^{2}-\left|\left\langle\phi_{+1} \mid \phi_{\downarrow}\right\rangle\right|^{2}$ represents the precision of the measurement (see more details in Methods). The quality factor $F$ and the precision $G$ are determined solely by the pointer states and satisfy the trade-off relation $F^{2}+G^{2} \leq 1 .^{18}$ A weak measurement is optimal if $F^{2}+G^{2}=1$ is satisfied.

Modified Bell test with weak measurement

In a typical Bell test scenario, one pair of entangled spin-1/2 particles is distributed between two separated observers, Alice and Bob (Fig. 1a), who each receive a binary input $x, y \in\{0,1\}$ and subsequently give a binary output $a, b \in\{1,-1\}$. For each input $x$ (y), Alice (Bob) performs a strong projective measurement of her (his) spin along a specific direction and obtains the outcome $a(b)$. The scenario is characterized by a joint probability distribution $P$ $(a b \mid x y)$ of obtaining outcomes $a$ and $b$, conditioned on measurement inputs $x$ for Alice and $y$ for Bob. The fixed measurement inputs $x$ and $y$ defines the correlations $C_{(x, y)}=\sum_{a, b} a b P(a b \mid x y)$. The $\mathrm{CHSH}$-Bell test is focused on the so-called $S$ value defined by the combination of correlations

$S=\left|C_{(0,0)}+C_{(0,1)}+C_{(1,0)}-C_{(1,1)}\right|$.

Whereas $S \leq 2$ in any local hidden variable theory, ${ }^{4}$ quantum theory gives a more relaxed bound of $2 \sqrt{2} .^{31}$

Here, we consider a new Bell scenario in which there are two observers Bob1 and Bob2 access to the same one-half of the entangled state of spin-1/2 particles (Fig. 1b). Alice, Bob1 and Bob2 each receive a binary input $x, y_{1}, y_{2} \in\{0,1\}$ and subsequently provide a binary output $a, b_{1}, b_{2} \in\{1,-1\}$. For each input $y_{1}$, Bob1 performs weak measurement of his spin along a specific direction, whereas Alice and Bob2 perform strong projective measurements for their input $x$ and $y_{2}$. With the outcome $b_{1}$, Bob1 sends the measured spin particle to Bob2. The scenario is now characterized by joint conditional probabilities $P\left(a b_{1} b_{2} \mid x y_{1} y_{2}\right)$, and an incisive question is raised whether Bob1 and Bob2 can both share nonlocality with Alice. The answer is surprisingly positive that the statistics of both Alice-Bob1 and Alice-Bob2 can indeed violate the $\mathrm{CHSH}-$ Bell inequality simultaneously. ${ }^{18}$

The quantities $G$ and $F$ of weak measurement, respectively, determine the $S$ values of Alice-Bob1 and Alice-Bob2 in the new Bell scenario. In the case that the Tsirelson's bound $2 \sqrt{2}$ of the $\mathrm{CHSH}-$ Bell inequality can be attained, the calculation gives (see more details in Methods)

$S_{A-B 1}=2 \sqrt{2} G, S_{A-B 2}=\sqrt{2}(1+F)$.

Realization of optimal weak measurement in a photonic system To observe significant double violations of the CHSH-Bell inequality, the realization of optimal weak measurement is a key and necessary requirement. In the original scheme proposed in ref. ${ }^{18}$ the spatial degree of freedom of particle is used as the pointer. However, the particle with common used spatial distributions, e.g. Gaussian distribution, only realizes sub-optimal weak measurement, i.e., $F^{2}+G^{2}<1$. Here, we propose and realize optimal weak measurement in a photonic system by using discrete pointer, i.e., path degree of freedom of photons instead of continuous pointer. ${ }^{32}$ It should be noted here that whether or not the pointer is continuous or discrete do not change any results discussed above.

Before illustration of the experimental realization, it should be emphasized first that weak measurement is mathematically equivalent to positive operator valued measures (POVMs) formalism ${ }^{33}$ and this becomes our basis of experimental design. For the spin system discussed above, if Bob1 performs weak measurement and obtains outcome \pm 1 , the states of measured system will accordingly collapse into

$\left|\Psi_{ \pm 1}\right\rangle_{s}=a\left\langle\phi_{ \pm 1} \mid \phi_{\uparrow}\right\rangle|\uparrow\rangle+\beta\left\langle\phi_{ \pm 1} \mid \phi_{\downarrow}\right\rangle|\downarrow\rangle$

with probability $P( \pm 1)=\operatorname{Tr}\left(\left|\Psi_{ \pm 1}\right\rangle_{s}\left\langle\Psi_{ \pm 1}\right|\right)$. The weak measurement of Bob1 is actually to realize a two-outcome POVMs with Kraus operators $^{34}$

$M_{ \pm 1}=\left\langle\phi_{ \pm 1} \mid \phi_{\uparrow}\right\rangle|\uparrow\rangle\left\langle\uparrow\left|+\left\langle\phi_{ \pm 1} \mid \phi_{\downarrow}\right\rangle\right| \downarrow\right\rangle\langle\downarrow|$

corresponding to outcome \pm 1 .

In our realization of weak measurement of Bob1 with photonic elements as shown in Fig. 2a, the measured photons are in polarization state and the path degree of freedom of photons is 

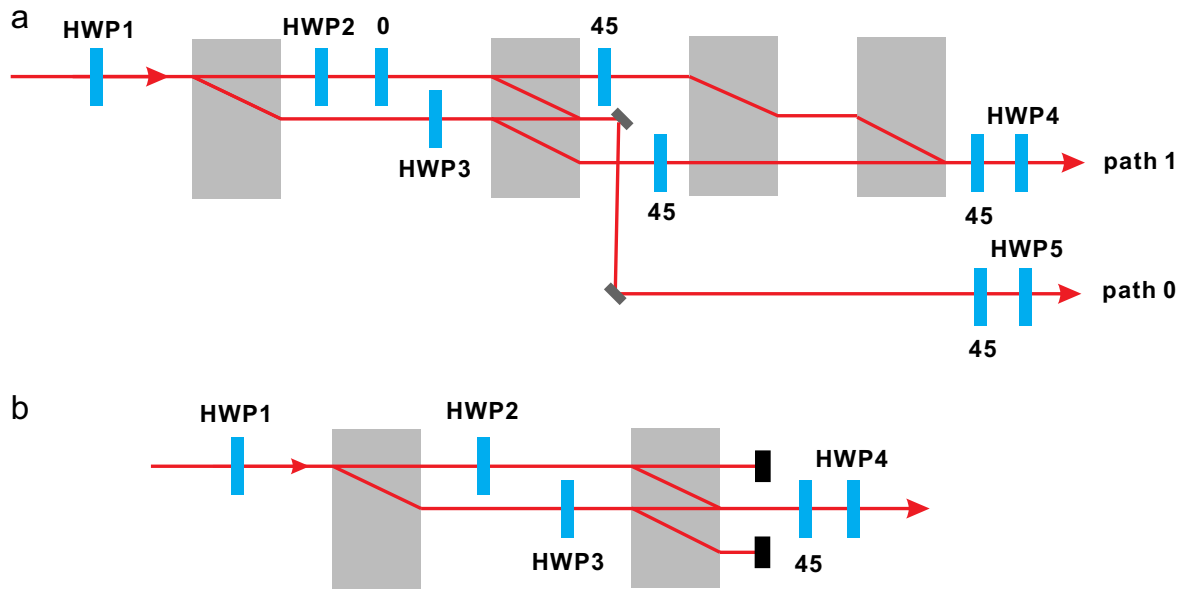

Fig. 2 Optimal weak measurement realized in a photonic system. a HWP2 and HWP3 are rotated at $\theta / 2$ and $\pi / 4-\theta / 2$ degree determining the strength of measurement $F=\sin 2 \theta$. Photons with vertical polarization state $|V\rangle$ transmit calcite beam displacer (BD) without change of its path while photons with horizontal polarization state $|H\rangle$ suffer a shift away from its original path. HWP1, HWP4 and HWP5 are rotated at the same degree $\varphi / 2$ to realize weak measurement of polarization observable $\sigma_{\varphi}=|\varphi\rangle\left\langle\varphi|-| \varphi^{\perp}\right\rangle\left\langle\varphi^{\perp}\right|$. The measurement outcome $+1(-1)$ is encoded in path $0(1)$ separately. $\mathbf{b}$ The setup, used in actual experiment, realizes same optimal weak measurement as shown above. The only difference is that specific outcome $+1(-1)$ can be selected by rotating HWP1 and HWP4. In the measurement of observation $\sigma_{\varphi}$ with HWP1 and HWP4 rotated at $\varphi / 2$ degree, outcome +1 is obtained when photons comes out of the setup and outcome -1 is obtained when HWP1 and HWP4 rotated at $\varphi / 2+\pi / 4$. Note that measurement outcome values are extracted in the final coincidence detection

used as pointer. In order to perform weak measurement in specific polarization basis $\left\{|\varphi\rangle,\left|\varphi^{\perp}\right\rangle\right\}$ with defined observable $\sigma_{\varphi}=|\varphi\rangle\langle\varphi|$ $-\left|\varphi^{\perp}\right\rangle\left\langle\varphi^{\perp}\right|$, we first transform the measured basis $\left\{|\varphi\rangle,\left|\varphi^{\perp}\right\rangle\right\}$ to basis $\{|H\rangle,|V\rangle\}$ via half wave plate (HWP1), then realize weak measurement of observable $\sigma_{H}=|H\rangle\langle H|-| V\rangle\langle V|$ via optical elements between HWP1 and HWP4, HWP5 and finally transform back to $\left\{|\varphi\rangle,\left|\varphi^{\perp}\right\rangle\right\}$ basis via HWP4 and HWP5. HWP1, HWP4 and HWP5 are rotated by the same angle $\varphi / 2$.

The key part of our setup is the realization of weak measurement of observable $\sigma_{H}$ and this is achieved by interference between calcite beam displacers (BDs) (Fig. 2). Consider photons with polarization state $|\Phi\rangle=\alpha|H\rangle+\beta|V\rangle$ to be measured, after interaction, the composite state of photons becomes $|\psi\rangle=a|H\rangle\left|\phi_{H}\right\rangle+\beta|V\rangle\left|\phi_{V}\right\rangle$ with $\left|\phi_{H}\right\rangle\left(\left|\phi_{V}\right\rangle\right)$ is the corresponding pointer state. The reading states $\left\{\left|\phi_{+1}\right\rangle,\left|\phi_{-1}\right\rangle\right\}$ in our realization are chosen as states of two separated paths 0 and 1 (Fig. 2a) denoted by $|0\rangle$ and $|1\rangle$. By rotating HWP2 and HWP3 between $\mathrm{BDs}$ at $\theta / 2$ and $\pi / 4-\theta / 2$ degrees respectively, the pointer states become

$$
\begin{aligned}
\left|\phi_{H}\right\rangle & =\cos \theta|0\rangle+\sin \theta|1\rangle, \\
\left|\phi_{V}\right\rangle & =\sin \theta|0\rangle+\cos \theta|1\rangle
\end{aligned}
$$

with $0 \leq \theta \leq \pi / 2$. The quality factor and information gain in our case are $F=\left\langle\phi_{H} \mid \phi_{V}\right\rangle=\sin 2 \theta$ and $G=1-\left|\left\langle 1 \mid \phi_{H}\right\rangle\right|^{2}-\left|\left\langle 0 \mid \phi_{V}\right\rangle\right|^{2}=$ $\cos 2 \theta$. The condition of optimal weak measurement $F^{2}+G^{2}=1$ is satisfied.

In practical experiment, we use the setup shown in Fig. $2 b$ instead of that shown in Fig. 2a. The setup shown in Fig. $2 \mathrm{~b}$ can realize the same optimal weak measurement as that in Fig. 2a and the only difference is that specific outcome can be selected by rotating HWP1 and HWP4. When Bob1 performs weak measurement of observable $\sigma_{\varphi}$ with HWP1 and HWP4 rotated at $\varphi / 2$ (or $\pi /$ $4-\varphi / 2)$ degree, photons comes out of setup have state $\left|\Psi_{+1}\right\rangle=$ $M_{+1}|\Phi\rangle$ (or $\left|\Psi_{-1}\right\rangle=M_{-1}|\Phi\rangle$ ) corresponding to outcome +1 (or $-1)$. Here, $M_{+1}=\cos \theta|\varphi\rangle\left\langle\varphi|-\sin \theta| \varphi^{\perp}\right\rangle\left\langle\varphi^{\perp}\left|, M_{-1}=\sin \theta\right| \varphi\right\rangle\langle\varphi|-$ $\cos \theta\left|\varphi^{\perp}\right\rangle\left\langle\varphi^{\perp}\right|$ are Kraus operators and Bob1 extracts his measurement outcomes by final coincidence detection given that the rotation angles of HWP1 and HWP4 are known to him. It should be emphasized here that the outcomes of Bob1 are actually obtained by Bob2 in our photonic experiment. This is because that the measurement of Bob1 is realized by coupling polarization of photons to its path and the outcomes are encoded in the path after measurement.

Experimental observation of double Bell inequality violations

In our Bell test experiment (Fig. 3), polarization-entangled pairs of photons in state $(|H\rangle|V\rangle-|V\rangle|H\rangle) / \sqrt{2}$ are generated by pumping a type-II apodized periodically poled potassium titanyl phosphate (PPKTP) crystal to produce photon pairs at a wavelength of $798 \mathrm{~nm}$. A $4.5 \mathrm{~mW}$ pump laser centered at a wavelength of $399 \mathrm{~nm}$ is produced by a Moglabs ECD004 laser, and a PPKTP crystal is embedded in the middle of a Sagnac interferometer to ensure the production of high-quality, high-brightness entangled pair. ${ }^{35,36}$ The maximum coincidence counting rates in the horizontal/ vertical basis are $\sim 3200 \mathrm{~s}^{-1}$. The visibility of coincidence detection for the maximally entangled state is measured to be $0.997 \pm 0.006$ in the horizontal/vertical polarization basis $\{|H\rangle,|V\rangle\}$ and $0.993 \pm$ 0.008 in the diagonal/antidiagonal polarization basis $\{(|H\rangle \pm|V\rangle) / \sqrt{2}\}$, achieved by rotating the polarization analyzers for two photons.

Alice, Bob1 and Bob2 each have two measurement choices, and for each choice, two trials are needed, corresponding to two different outcomes. For each fixed $\theta$, which determines the strength of the weak measurement $F=\sin 2 \theta$, we have implemented 64 trials for calculating $S_{A-B 1}$ and $S_{A-B 2}$. To ensure that the Tsirelson's bound $2 \sqrt{2}$ can be approached, Alice chooses measurement along direction $Z$ or $X$, while Bobs choose measurement along $(-Z+X) / \sqrt{2}$ or $-(Z+X) / \sqrt{2}$ direction. In this experiment, HWP6 is set at $\left(0^{\circ}, 45^{\circ}\right)$ or $\left(22.5^{\circ}, 67.5^{\circ}\right)$, corresponding to Alice's measurement along the $Z$ or $X$ direction, while HWP1 and HWP5, representing measurements of Bob1 and Bob2, are set at $\left(-11.25^{\circ}, 33.75^{\circ}\right)$ or $\left(11.25^{\circ}, 56.25^{\circ}\right)$, corresponding to the $(-Z+X) / \sqrt{2}$ or $-(Z+X) / \sqrt{2}$ direction, respectively. For instance, if HWP1, HWP4 and HWP5 are rotated at $-11.25^{\circ}$ and HWP6 is fixed at $0^{\circ}$, the three-variable joint conditional probability $P\left[a=1, b_{1}=1, b_{2}=1 \mid x=Z, y_{1}=(-Z+X) / \sqrt{2}, y_{2}=\right.$ $(-Z+X) / \sqrt{2}]$ is obtained by the final coincidence detection. The other joint conditional probabilities can be detected via similar various combination of HWP1, HWP4, HWP5 and HWP6.

Five different angles $\theta=\left\{4^{\circ}, 16.4^{\circ}, 18.4^{\circ}, 20.5^{\circ}, 28^{\circ}\right\}$ are chosen from which the values of $\theta=\left\{16.4^{\circ}, 18.4^{\circ}, 20.5^{\circ}\right\}$ are located in the region where double violations are predicted to be observed. In 


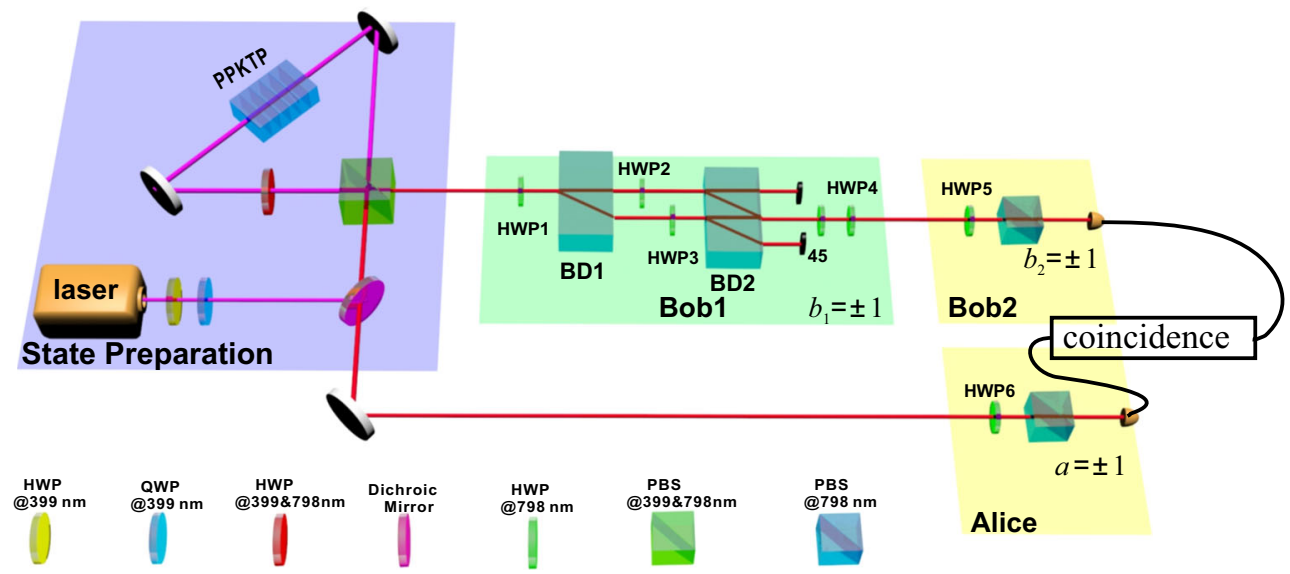

Fig. 3 Measurement setup. Polarization-entangled pairs of photons are produced by pumping a type-ll apodized periodically poled potassium titanyl phosphate (PPKTP) crystal placed in the middle of a Sagnac-loop interferometer with dimensions of $1 \mathrm{~mm} \times 2 \mathrm{~mm} \times 20 \mathrm{~mm}$ and with end faces with anti-reflective coating at wavelengths of $399 \mathrm{~nm}$ and $798 \mathrm{~nm}$. The photon emitted to Alice is measured via a combination of HWP6 and PBS. The green area shows the weak disturbance measurement setup of Bob1. During the experiment, HWP2, HWP3 are rotated by $\theta / 2, \pi / 4-\theta / 2$ according to the experimental requirement. HWP1 is used for Bob1's measurement, and HWP4 is rotated by the same angle as HWP1 to transform the photons polarization state back to the measurement basis after the photon passes through two beam displacers (BDs). The photon passing through HWP4 is then sent to Bob2 for a strong projective measurement with HWP5 and PBS. In the final stage, two-photon coincidences at $6 \mathrm{~s}$ are recorded by avalanche photodiode single-photon detectors and a coincidence counter (ID800)

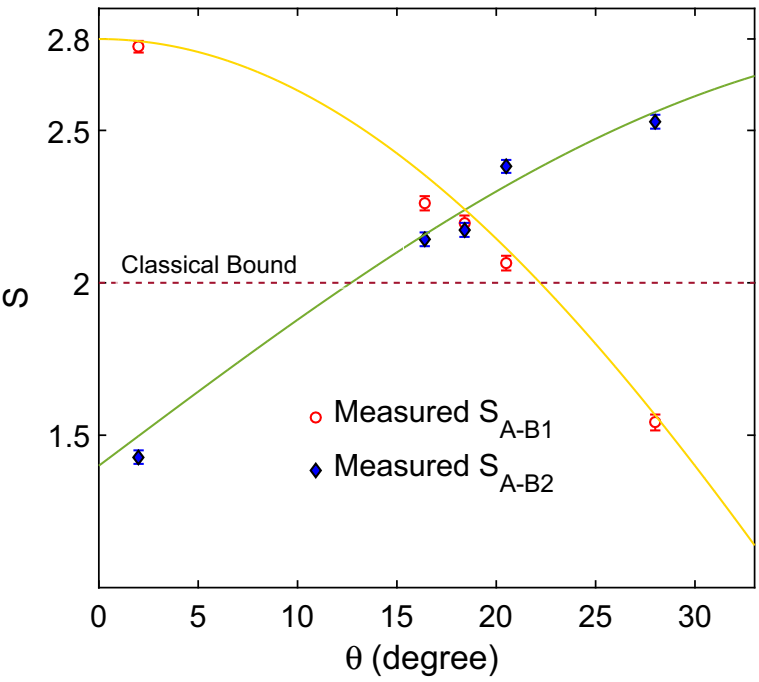

Fig. 4 Experimental results. The yellow and green curves represent the theoretical predictions for $S_{A-B 1}$ and $S_{A-B 2}$, respectively, whereas the red circles and blue rhombus indicate the practical measured results for $S_{A-B 1}$ and $S_{A-B 2}$, corresponding $\theta=\left\{4^{\circ}, 16.4^{\circ}, 18.4^{\circ}, 20.5^{\circ}\right.$, $\left.28^{\circ}\right\}$. Double violations are observed in $\theta=\left\{16.4^{\circ}, 18.4^{\circ}, 20.5^{\circ}\right\}$ with $\sim 10$ standard deviations. The error bars are calculated according to Poissonian counting statistics

particular, the balanced double violations $S_{A-B 1}=S_{A-B 2}=2.26$ are presented under optimal weak measurement when $F=0.6$, corresponding to $\theta=18.4^{\circ}$. Our final results are shown in Fig. 4, where double violations are clearly displayed at $\theta=\left\{16.4^{\circ}, 18.4^{\circ}\right.$, $\left.20.5^{\circ}\right\}$ with $\sim 10$ standard deviations. Specifically, when $\theta=18.4^{\circ}$ we obtain $S_{A-B 1}=2.20 \pm 0.02$ and $S_{A-B 2}=2.17 \pm 0.02$. Considering the possible statistical error, systematic error and imperfection of our apparatus, these experimental results fit well within the theoretical predictions.

\section{DISCUSSION}

In conclusion, we have observed double violations of the $\mathrm{CHSH}$ Bell inequality for the entangled state of photon pairs by using a strength continuous-tunable optimal weak measurement. Our experimental results verify the non-locality distribution among multiple observers and shed new light on our understanding of the fascinating properties of non-locality and quantum measurement. The weak measurement technique used herein can find significant applications in unbounded randomness certification, ${ }^{19,20}$ which is a valuable resource applied from quantum cryptography $^{37,38}$ and quantum gambling ${ }^{39,40}$ to quantum simulation. ${ }^{41}$ Here, the $S$ value of the correlation between Alice and Bob2 is determined by the quality factor of Bob1's weak measurement, implying that Bob1 can control the non-local correlation of Alice and Bob2 by manipulating the strength of his measurement. This result provides tremendous motivation for the further quantum steering research. ${ }^{21,22}$

Note added. After we have posted this work online we noticed that double Bell inequality violations was also observed using similar method in ref. ${ }^{42}$ though they did not realize optimal weak measurement.

\section{METHODS}

Weak measurement on a spin-1/2 particles

Consider a typical measurement of spin observable $\sigma_{z}$ of a spin-1/2 particle with eigenstates satisfy $\sigma_{z}|\uparrow\rangle=|\uparrow\rangle$ and $\sigma_{z}|\downarrow\rangle=-|\downarrow\rangle$. The initial pointer state $|\phi\rangle$ is entangled with spin system after measurement interaction

$$
\begin{aligned}
\hat{U}(\varrho \otimes|\phi\rangle\langle\phi|) \hat{U}^{\dagger}= & \pi_{\uparrow} \varrho \pi_{\uparrow} \otimes\left|\phi_{\uparrow}\right\rangle\left\langle\phi_{\uparrow}\left|+\pi_{\downarrow} \varrho \pi_{\downarrow} \otimes\right| \phi_{\downarrow}\right\rangle\left\langle\phi_{\downarrow}\right| \\
& +\pi_{\uparrow} \varrho \pi_{\downarrow} \otimes\left|\phi_{\uparrow}\right\rangle\left\langle\phi_{\downarrow}\left|+\pi_{\downarrow} \varrho \pi_{\uparrow} \otimes\right| \phi_{\downarrow}\right\rangle\left\langle\phi_{\uparrow}\right|,
\end{aligned}
$$

where $\varrho$ represents the initial state of spin system, $\pi_{\uparrow} \equiv|\uparrow\rangle\left\langle\uparrow\left|, \pi_{\downarrow} \equiv\right| \downarrow\right\rangle\langle\downarrow|$ are the projectors on the eigenstates of $\sigma_{z}$ and $\left|\phi_{\uparrow}\right\rangle,\left|\phi_{\downarrow}\right\rangle$ are the evolved pointer states corresponding to $|\uparrow\rangle,|\downarrow\rangle$, respectively.

The state of spin system, after tracing out pointer, becomes

$\rho=\pi_{\uparrow} \varrho \pi_{\uparrow}+\pi_{\downarrow} \varrho \pi_{\downarrow}+\pi_{\uparrow} \varrho \pi_{\downarrow} \otimes\left\langle\phi_{\downarrow} \mid \phi_{\uparrow}\right\rangle+\pi_{\downarrow} \varrho \pi_{\uparrow} \otimes\left\langle\phi_{\uparrow} \mid \phi_{\downarrow}\right\rangle$.

To quantify the disturbance of measurement to the spin system, a quantity $F$ called quality factor of measurement can be defined ${ }^{18}$

$F \equiv\left\langle\phi_{\uparrow} \mid \phi_{\downarrow}\right\rangle$.

Usually, $F$ is a complex value. Without loss of generality, here we take it as a real number for the simplicity of discussion. The Eq. (9) thus can be 
reformulated as

$\rho=F Q+(1-F)\left(\pi_{\uparrow} \varrho \pi_{\uparrow}+\pi_{\downarrow} \varrho \pi_{\downarrow}\right)$

as that $\rho=\pi_{\uparrow} \rho \pi_{\uparrow}+\pi_{\downarrow} \rho \pi_{\downarrow}+\pi_{\uparrow} \rho \pi_{\downarrow}+\pi_{\downarrow} \rho \pi_{\uparrow}$.

If $F=0$, the spin-up state $|\uparrow\rangle$ and spin-down state $|\downarrow\rangle$ can be distinguished definitely through the orthogonal pointer states $\left|\phi_{\uparrow}\right\rangle$ and $\mid$ $\left.\phi_{\downarrow}\right\rangle$. The state of spin system $\rho$ is thus reduced to a state of completely decohered in the eigenbasis of $\sigma_{z}$ and the measurement in this case is called a strong measurement in which we can obtain the maximum information about the system. There is no measurement at all if $F=1$, i.e., the pointer state is the same for spin up or spin down. The measurement is called weak when $F \in(0,1)$ in which we can obtain partial information of the spin states with partial disturbance on it. It is obvious from Eq. (11) that a weak measurement can be considered as the combination of a strong measurement and none of measurement operationally. The quality factor $F$ thus reflects the strength of measurement and the disturbance of measurement.

The measurement of a spin-1/2 system only have two outcomes +1 and -1 corresponding to eigenstates $|\uparrow\rangle$ and $|\downarrow\rangle$, respectively. If the measurement is strong, the probability of obtaining outcome +1 (or -1 ) can be easily determined by the Born rule that $P(+1)=\operatorname{Tr}\left(\pi_{\uparrow} \rho\right)$ (or $P(-1)=$ $\left.\operatorname{Tr}\left(\pi_{\downarrow} \rho\right)\right)$. However, in weak measurement the non-orthogonality of pointer states $F=\left\langle\phi_{\uparrow} \mid \phi_{\downarrow}\right\rangle \neq 0$ brings the uncertainty ambiguity. In practice, an observer, who performs weak measurement, has to choose a complete orthogonal set of pointer states $\left|\phi_{+1}\right\rangle,\left|\phi_{-1}\right\rangle$ as reading states of pointer to define outcomes $+1,-1$ corresponding to the spin eigenstates $|\uparrow\rangle,|\downarrow\rangle$. To calculate the probability of outcome +1 (or -1 ), the state of pointer after measurement have to be considered. Tracing out the spin degree of freedom in Eq. (8), we obtain the post-measurement state of the pointer

$\rho_{p}=\operatorname{Tr}\left(\pi_{\uparrow} \varrho\right) \otimes\left|\phi_{\uparrow}\right\rangle\left\langle\phi_{\uparrow}\left|+\operatorname{Tr}\left(\pi_{\downarrow} \varrho\right) \otimes\right| \phi_{\downarrow}\right\rangle\left\langle\phi_{\downarrow}\right|$.

The probability of outcome +1 (or -1 ) now becomes the probability of obtaining the state $\left|\phi_{+1}\right\rangle$ (or $\left|\phi_{-1}\right\rangle$ ) of pointer. The probability of outcome \pm 1 becomes

$P( \pm 1)=\operatorname{Tr}\left(\pi_{\uparrow} \varrho\right)\left|\left\langle\phi_{ \pm 1} \mid \phi_{\uparrow}\right\rangle\right|^{2}+\operatorname{Tr}\left(\pi_{\downarrow} \varrho\right)\left|\left\langle\phi_{ \pm 1} \mid \phi_{\downarrow}\right\rangle\right|^{2}$.

If the pointer states are considered in position representation, the observer can associate positive positions to outcome +1 and negative positions to outcome -1 . In general, the reading states of pointer $\left|\phi_{+1}\right\rangle$, $\left.\phi_{-1}\right\rangle$ should be chosen that the maximum of $\left|\left\langle\phi_{+1} \mid \phi_{\uparrow}\right\rangle\right|^{2}$ and $\left|\left\langle\phi_{-1} \mid \phi_{\downarrow}\right\rangle\right|^{2}$ are achieved. If the reading states ensure that $\left|\left\langle\phi_{+1} \mid \phi_{\uparrow}\right\rangle\right|^{2}=\left|\left\langle\phi_{-1} \mid \phi_{\downarrow}\right\rangle\right|^{2}$, i.e., the probabilities of obtaining correct outcomes are equal for spin up and spin down, the measurement is called unbiased. Here we only focus on unbiased measurement and assume that the reading states satisfy conditions $\left|\left\langle\phi_{+1} \mid \phi_{\uparrow}\right\rangle\right|^{2}=\left|\left\langle\phi_{-1} \mid \phi_{\downarrow}\right\rangle\right|^{2}$ and $\left|\left\langle\phi_{+1} \mid \phi_{\downarrow}\right\rangle\right|^{2}=\left|\left\langle\phi_{-1} \mid \phi_{\uparrow}\right\rangle\right|^{2}$. In this case, the probability $P( \pm 1)$ can be reformulated as

$P( \pm 1)=G \cdot \frac{1}{2}\left[1 \pm \operatorname{Tr}\left(\sigma_{z} \varrho\right)\right]+(1-G) \cdot \frac{1}{2}$,

where $\sigma_{z}=\pi_{\uparrow}-\pi_{\downarrow}$ is the spin observable and $G=1-\left|\left\langle\phi_{+1} \mid \phi_{\downarrow}\right\rangle\right|^{2}-\mid\left\langle\phi_{-1}\right|$ $\left.\phi_{\uparrow}\right\rangle\left.\right|^{2}=1-2\left|\left\langle\phi_{+1} \mid \phi_{\downarrow}\right\rangle\right|^{2}$ defines the precision of measurement. The precision of measurement $G$ indicates the correctness or the extent of unambiguity of outcomes.

The state of the spin system, after the observer obtains outcome \pm 1 , becomes

$$
\begin{aligned}
\rho_{ \pm 1}= & \left\langle\phi_{ \pm 1}\left|\hat{U}_{\varrho} \otimes\right| \phi\right\rangle\left\langle\phi\left|\hat{U}^{\dagger}\right| \phi_{ \pm 1}\right\rangle \\
= & \pi_{\uparrow} \varrho \pi_{\uparrow}\left|\left\langle\phi_{ \pm 1} \mid \phi_{\uparrow}\right\rangle\right|^{2}+\pi_{\downarrow} \varrho \pi_{\downarrow}\left|\left\langle\phi_{ \pm 1} \mid \varphi_{\downarrow}\right\rangle\right|^{2} \\
& \quad+\pi_{\uparrow} \varrho \pi_{\downarrow}\left\langle\phi_{ \pm 1} \mid \phi_{\uparrow}\right\rangle\left\langle\phi_{\downarrow} \mid \phi_{ \pm 1}\right\rangle+\pi_{\downarrow} \varrho \pi_{\uparrow}\left\langle\phi_{ \pm 1} \mid \phi_{\downarrow}\right\rangle\left\langle\phi_{\uparrow} \mid \phi_{ \pm 1}\right\rangle .
\end{aligned}
$$

Since that $\quad\left|\left\langle\phi_{+1} \mid \phi_{\uparrow}\right\rangle\right|^{2}=\frac{1+G}{2},\left|\left\langle\phi_{+1} \mid \phi_{\downarrow}\right\rangle\right|^{2}=\frac{1-G}{2} \quad$ and $\left\langle\phi_{+1} \mid \phi_{\downarrow}\right\rangle\left\langle\phi_{\uparrow} \mid \phi_{+1}\right\rangle=\left\langle\phi_{+1} \mid \phi_{\uparrow}\right\rangle\left\langle\phi_{\downarrow} \mid \phi_{+1}\right\rangle=\frac{F^{2}}{2}$, Eq. (15) can be reformulated as (unnormalized)

$\rho_{ \pm 1}=\frac{F}{2} \varrho+\frac{1 \pm G-F}{2} \pi_{\uparrow} \varrho \pi_{\uparrow}+\frac{1 \mp G-F}{2} \pi_{\downarrow} \varrho \pi_{\downarrow}$.

The quality factor $F$ and the precision $G$ satisfy the trade-off relation ${ }^{18}$

$F^{2}+G^{2} \leq 1$

A weak measurement is optimal if $F^{2}+G^{2}=1$, otherwise it is suboptimal.
Relation between weak measurement and Bell non-locality

The connection between weak measurement and non-locality can be shown in the new Bell scenario that one pair of entangled spin- $\frac{1}{2}$ particles are delivered to Alice, Bob1 and Bob2, here Bob1 and Bob2 access to the same particle shown in Fig. 1 of main text. Contrary to Bob2 who performs the strong measurement, Bob1 performs the weak measurement before Bob2. After the measurement of Bob1, the particle will be sent to Bob2 who has no idea of Bob1's existence. Suppose that the entangled pair is in the singlet state

$|\Psi\rangle=\frac{1}{\sqrt{2}}(|\uparrow\rangle|\downarrow\rangle-|\downarrow\rangle|\uparrow\rangle)$.

Similar to the standard Bell scenario, Alice, Bob1 and Bob2 each receives a binary input $x_{1} y_{1}, y_{2} \in\{0,1\}$ and accordingly performs measurement of their spin along the corresponding direction $\vec{\lambda}_{x}, \vec{\mu}_{y_{1}}, \vec{\nu}_{y_{2}}$ respectively. The outcomes of their measurement are labelled by $a, b_{1}, b_{2} \in\{+1,-1\}$. To study correlations between Alice and Bobs, we need to calculate the conditional probability distributions $P\left(a b_{1} b_{2} \mid x y_{1} y_{2}\right)$ that can be simplified by using no-signalling condition

$P\left(a b_{1} b_{2} \mid x y_{1} y_{2}\right)=P(a \mid x) P\left(b_{1} \mid x y_{1} a\right) P\left(b_{2} \mid x y_{1} y_{2} a b_{1}\right)$.

The probability of obtaining outcome $a$ conditioned on $x$ for Alice can be easily shown to be $P(a \mid x)=\frac{1}{2}$ as that any strong measurement on onehalf of singlet state gives outcomes with equal probability. After the measurement of Alice, the spin state of another particle sent to Bob1 will collapse into the state that in an opposite spin direction with respect to Alice's post-measurement state

$\rho_{\mid x a}=\pi_{-a \vec{\lambda}_{x}}=\frac{1}{2}\left(I-a \vec{\lambda}_{x} \cdot \vec{\sigma}\right)$,

where $\pi_{-a \vec{\lambda}_{x}}$ represents the spin projector along the direction $-a \vec{\lambda}_{x}$ and $I, \vec{\sigma}$ are identity operator and Pauli operator, respectively. The measurement of Bob1 is weak, the probability $P\left(b_{1} \mid x y_{1} a\right)$ is determined by Eq. (14) and

$$
\begin{aligned}
P\left(b_{1} \mid x y_{1} a\right) & =G \cdot \operatorname{Tr}\left(\pi_{b_{1} \vec{\mu}_{y_{1}}} \rho_{\mid x a}\right)+(1-G) \cdot \frac{1}{2} \\
& =\frac{1-G a b_{1} \vec{\lambda}_{x} \cdot \vec{\mu}_{y_{1}}}{2},
\end{aligned}
$$

where $\operatorname{Tr}\left(\pi_{b_{1} \vec{\mu}_{y_{1}}} \rho_{\mid x a}\right)=\frac{1-a b_{1} \vec{\lambda}_{x} \cdot \vec{\mu}_{y_{1}}}{2}$ and $G$ is the precision of Bob1's weak measurement. The spin state of Bob1's particle after weak measurement, according to Eq. (16), becomes

$$
\begin{array}{r}
\rho_{\mid x y_{1} a b_{1}}=\frac{F}{2} \rho_{\mid x a}+\frac{1+b_{1} G-F}{2} \pi_{\vec{\mu}_{y_{1}}} \rho_{\mid x a} \pi_{\vec{\mu}_{y_{1}}} \\
+\frac{1-b_{1} G-F}{2} \pi_{-\vec{\mu}_{y_{1}}} \rho_{\mid x a} \pi_{-\vec{\mu}_{y_{1}}}
\end{array}
$$

with its norm trace $\operatorname{Tr}\left(\rho_{\mid x y_{1} a b_{1}}\right)=P\left(b_{1} \mid x y_{1} a\right)$ and $F$ is quality factor of the weak measurement. The probability of obtaining outcome $b_{2}$ for Bob2's strong measurement is

$$
\begin{aligned}
P\left(b_{2} \mid x y_{1} y_{2} a b_{1}\right)= & \operatorname{Tr}\left(\pi_{b_{2} \vec{\nu}_{y_{2}}} \tilde{\rho}_{\mid x y_{1} a b_{1}}\right) \\
= & \frac{1}{P\left(b_{1} \mid x y_{1} a\right)}\left\{\frac{F}{4}\left(1-a b_{2} \vec{\lambda}_{x} \cdot \vec{\nu}_{y_{2}}\right)\right. \\
& \quad+\frac{1-F}{4}\left[1-a b_{2}\left(\vec{\lambda}_{x} \cdot \vec{\mu}_{y_{1}}\right)\left(\vec{\mu}_{y_{1}} \cdot \vec{\nu}_{y_{2}}\right)\right] \\
& \left.+\frac{b_{1} G}{4}\left(b_{2} \vec{\mu}_{y_{1}} \cdot \vec{\nu}_{y_{2}}-a \vec{\lambda}_{x} \cdot \vec{\mu}_{y_{1}}\right)\right\},
\end{aligned}
$$

where $\tilde{\rho}_{\mid x y_{1} a b_{1}}=\frac{1}{P\left(b_{1} \mid x y_{1} a\right)} \rho_{\mid x y_{1} a b_{1}}$ is a normalized state.

Now we can calculate conditional probabilities $P\left(a b_{1} b_{2} \mid x y_{1} y_{2}\right)$ according to Eq. (12)

$$
\begin{aligned}
P\left(a b_{1} b_{2} \mid x y_{1} y_{2}\right)=\frac{F}{4} & \left(\frac{1-a b_{2} \vec{\lambda}_{x} \cdot \vec{\nu}_{y_{2}}}{2}\right) \\
& +\frac{1-F}{4}\left[\frac{1-a b_{2}\left(\vec{\lambda}_{x} \cdot \vec{\mu}_{y_{1}}\right)\left(\vec{\mu}_{y_{1}} \cdot \vec{\nu}_{y_{2}}\right)}{2}\right] \\
& -\frac{b_{1} G}{4}\left(\frac{a \vec{\lambda}_{x} \cdot \vec{\mu}_{y_{1}}-b_{2} \vec{\mu}_{y_{1}} \cdot \vec{\nu}_{y_{2}}}{2}\right) .
\end{aligned}
$$

As probability lies between 0 and 1 , if $\vec{\lambda}_{0}=\vec{Z}, \vec{\mu}_{0}=-\vec{X}$ and $\vec{\nu}_{0}=$ $\vec{Z} \sin \theta-\vec{X} \cos \theta$ are chosen, we obtain

$P(1-11 \mid 000)=F \sin \theta+G \cos \theta \leq 1$

along with outcomes $a=b_{2}=1$ and $b_{1}=-1$. This is the expression of a tangent to the unit circle $F^{2}+G^{2}=1$ and obviously the optimal pointer saturates this constraint. 
The non-local correlation of Alice and Bobs's can be shown by calculating $S$ value defined as

$S_{\text {Alice-Bob }}=\left|C_{(0,0)}^{n}+C_{(0,1)}^{n}+C_{(1,0)}^{n}-C_{(1,1)}^{n}\right|$,

where $n \in\{1,2\}$ and $C_{\left(x, y_{n}\right)}^{n}$ defines correlation of Alice and Bob's measurement outcomes

$C_{\left(x, y_{n}\right)}^{n}=\operatorname{Tr}\left(\rho_{n} \sigma_{\vec{\lambda}_{x}} \otimes \sigma_{\vec{\mu}_{y_{n}}}\right)=\sum_{a, b_{n}} a b_{n} P\left(a b_{n} \mid x y_{n}\right)$

with $\rho_{n}$ is the state of the spin- $\frac{1}{2}$ entangled pair possessed by Alice and Bobs, $\sigma_{\vec{\lambda}_{x}}, \sigma_{\vec{\mu}_{\text {yn }}}$ represent the spin observables corresponding to directions $\vec{\lambda}_{x}$ and $\vec{\mu}_{y_{1}}$, respectively.

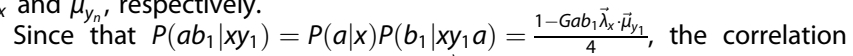
$C_{\left(x, y_{1}\right)}$ of Alice and Bob1 is $C_{\left(x, y_{1}\right)}=-G \vec{\lambda}_{x} \cdot \vec{\mu}_{y_{1}}$ and thus we have

$S_{A-B 1}=G \cdot\left|\vec{\lambda}_{0} \cdot \vec{\mu}_{0}+\vec{\lambda}_{0} \cdot \vec{\mu}_{1}+\vec{\lambda}_{1} \cdot \vec{\mu}_{0}-\vec{\lambda}_{1} \cdot \vec{\mu}_{1}\right|$.

Similarly

$$
\begin{aligned}
P\left(a b_{2} \mid x y_{2}\right)= & \sum_{b_{1}, y_{1}} P\left(a b_{1} b_{2} \mid x y_{1} y_{2}\right) \\
= & \frac{F}{4}\left(\frac{\left.1-a b_{2} \vec{\lambda}_{x} \cdot \vec{\nu}_{y_{2}}\right)}{2}\right) \\
& \left.\quad+\frac{1-F}{4} \sum_{y_{1}}\left[\frac{1-a b_{2}\left(\vec{\lambda}_{x} \cdot \vec{\mu}_{y_{1}}\right)\left(\vec{\mu}_{y_{1}} \cdot \vec{v}_{y_{2}}\right)}{2}\right)\right]
\end{aligned}
$$

and

$C_{\left(x, y_{2}\right)}=-\frac{F}{2} \vec{\lambda}_{x} \cdot \vec{\nu}_{y_{2}}-\frac{1-F}{2} \sum_{y_{1}}\left(\vec{\lambda}_{x} \cdot \vec{\mu}_{y_{1}}\right)\left(\vec{\mu}_{y_{1}} \cdot \vec{\nu}_{y_{2}}\right)$.

The $S$ value of Alice and Bob2 is calculated as

$$
\begin{aligned}
S_{A-B 2}=\mid \frac{F}{2}\left(\vec{\lambda}_{0} \cdot \vec{\nu}_{0}+\vec{\lambda}_{0} \cdot \vec{\nu}_{1}+\vec{\lambda}_{1} \cdot \vec{\nu}_{0}-\vec{\lambda}_{1} \cdot \vec{\nu}_{1}\right) \\
\quad-\frac{1-F}{2} \sum_{y_{1}}\left[\left(\vec{\lambda}_{0} \cdot \vec{\mu}_{y_{1}}\right)\left(\vec{\mu}_{y_{1}} \cdot \vec{\nu}_{0}\right)+\left(\vec{\lambda}_{0} \cdot \vec{\mu}_{y_{1}}\right)\left(\vec{\mu}_{y_{1}} \cdot \vec{\nu}_{1}\right)\right. \\
\left.+\left(\vec{\lambda}_{1} \cdot \vec{\mu}_{y_{1}}\right)\left(\vec{\mu}_{y_{1}} \cdot \vec{\nu}_{0}\right)-\left(\vec{\lambda}_{1} \cdot \vec{\mu}_{y_{1}}\right)\left(\vec{\mu}_{y_{1}} \cdot \vec{\nu}_{1}\right)\right] \mid .
\end{aligned}
$$

In the case that quantum bound $2 \sqrt{2}$ is obtained, i.e., Alice measures in the directions $\vec{Z}$ or $\vec{X}$ according to her inputs 0 or 1 , whereas Bob1 and Bob2 measure in the directions $\frac{-(\vec{Z}+\vec{X})}{\sqrt{2}}$ or $\frac{-\vec{Z}+\vec{X}}{\sqrt{2}}$ for their respective inputs 0 or 1 , we obtain

$$
\begin{aligned}
& S_{A-B 1}=2 \sqrt{2} G \\
& S_{A-B 2}=\sqrt{2}(1+F),
\end{aligned}
$$

which implies that the non-locality correlation of Alice and Bob1 and correlation of Alice and Bob2 are totally determined by the weak measurement performed by Bob1. Double violations happen when quality factor $F$ and precision $G$ of weak measurement satisfy $F \in(\sqrt{2}-1,1]$ and $G \in\left(\frac{\sqrt{2}}{2}, 1\right]$. Restricted by the general condition $F^{2}+G^{2} \leq 1$, the area that double violations exist is limited. In the case that optimal weak measurement is realized, which means $F^{2}+G^{2}=1$, double violations can be observed only when $F \in\left(\sqrt{2}-1, \frac{\sqrt{2}}{2}\right)$. If $F=0.6$ and $G=0.8$ are chosen, we obtain the optimal double violations $S_{\text {Alice-Bob1 }}=S_{\text {Alice-Bob2 }} \approx$ 2.26 .

Demonstration of entangled photons source in experiment In our experiment, high-quality polarization-entangled photon source is produced by pumping a type-ll apodized periodically poled potassium titanyl phosphate (PPKTP) crystal inside a Sagnac-loop interferometer. The PPKTP has dimensions of $1 \mathrm{~mm} \times 2 \mathrm{~mm} \times 20 \mathrm{~mm}$ and the end faces are anti-reflective coated at wavelengths of $399 \mathrm{~nm}$ and $798 \mathrm{~nm}$. The temperature of the crystal is controlled by using a home-made temperature controller with stability of $\pm 2 \mathrm{mK}$. The ultraviolet (UV) pump beam is generated from a commercial Moglabs ECD004 laser. One UV quarter wave plate (QWP) and one UV half wave plate (HWP) are placed at the input port of the interferometer for controlling the power and relative phase of pump beam inside the Sagnac-loop interferometer. Polarization orthogonal pump beams are separated by a dual wavelength polarized beam splitter (DPBS). The vertical polarized pump beam is rotated to horizontal polarization by using a dual wavelength HWP before interact with the PPKTP crystal for spontaneous parametric down-conversion

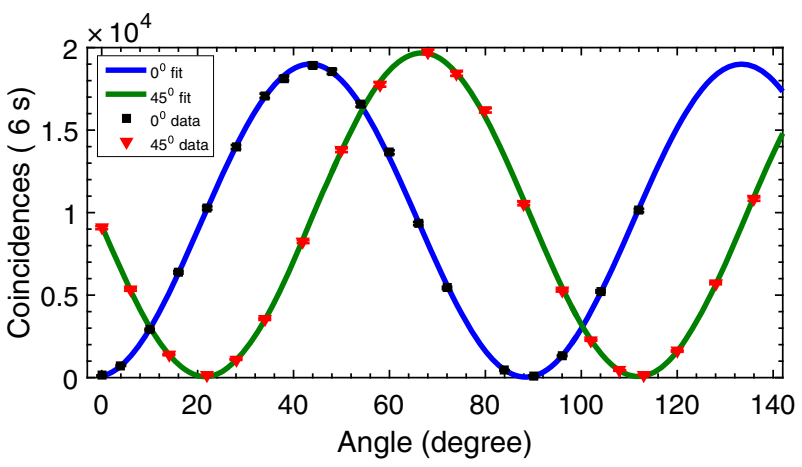

Fig. 5 Polarization interference results

(SPDC). Orthogonal polarized photon pairs are generated in two counter propagating directions combined at the DPBS. The photon emitted to Alice is first separated from the pump beam by using a dichromatic mirror (DM) and then measured projectively via combination of HWP and PBS by Alice. The photon sent to Bob, first passes through the weak measurement setup of Bob1 and is subsequently sent to Bob2 for projective measurement.

The state of photon pair output from the interferometer can be expressed as

$|\psi\rangle=\frac{1}{\sqrt{2}}\left(|H\rangle|V\rangle+e^{i \theta}|V\rangle|H\rangle\right)$,

where the relative phase $\vartheta$ is determined by the relative position of QWP and HWP at the input port. In our experiment, the phase $\vartheta$ is tuned to $\pi$ such that the singlet state is produced

$\left|\phi^{-}\right\rangle=\frac{1}{\sqrt{2}}(|H\rangle|V\rangle-|V\rangle|H\rangle)$.

The quality of our source is characterized by using a two-photon polarization interference shown in Fig. 5. The $399 \mathrm{~nm}$ wavelength pump beam's power is fixed at $4.5 \mathrm{~mW}$ and the coincidence windows is set in $2 \mathrm{~ns}$. The single counts in $6 \mathrm{~s}$ is about 375,000 and 275,000 and the maximum coincidence is about 19,000 . The raw visibilities in $0^{\circ} / 90^{\circ}$ and $45^{\circ}-45^{\circ}$ are $(99.70 \pm 0.06)$ percent and $(99.32 \pm 0.08)$ percent, respectively. Therefore high visibilities guarantee the large violation of Bell-CHSH inequality.

\section{DATA AVAILABILITY}

The data that support the findings of this study are available from the corresponding author upon request.

\section{ACKNOWLEDGEMENTS}

The authors thank Yun-Feng Huang, Bi-Heng Liu and Bao-Sen Shi for helpful discussions and technical supports. M.-J.H. also acknowledges Sheng Liu, Zhi-Bo Hou, Jian Wang, Chao Zhang, Ya Xiao, Dong-Sheng Ding, Yong-Nan Sun and Geng-Chen for many useful suggestions and stimulated discussions. This work was supported by National key R\&D program (No. 2016YFA0301300 and No. 2016YFA0301700), National Natural Science Foundation of China (No. 61275122, No. 11674306 and 61590932), Youth Innovation Promotion Association CAS and Anhui Initiative in Quantum Information Technologies.

\section{AUTHOR CONTRIBUTIONS}

Y.-S.Z. and M.-J.H. started the project and designed the experiment. Z.-Y.Z., M.-J.H. and X.-M.H. performed the experiment and completed the data analysis. M.-J.H., C.-F. L. and Y.-S.Z. provided the theoretical calculations. Y.-S.Z. and G.-C.G. supervised the project. M.-J.H. wrote the manuscript and all authors participated in discussions. M.-J. H. and Z.-Y.Z. have contributed equally to this work.

\section{ADDITIONAL INFORMATION}

Competing interests: The authors declare no competing interests.

Publisher's note: Springer Nature remains neutral with regard to jurisdictional claims in published maps and institutional affiliations. 


\section{REFERENCES}

1. Einstein, A., Podolsky, B. \& Rosen, N. Can quantum-mechanical description of physical reality be considered complete? Phys. Rev. Lett. 47, 777 (1935).

2. Bell, J. S. On the Einstein-Podolsky-Rosen paradox. Physics 1, 195-200 (1964).

3. Bell, J. S. Speakable and unspeakable in quantum mechanics: collected papers on quantum philosophy. (Cambridge University Press, London 2004).

4. Clauser, J. F., Horne, M. A., Shimony, A. \& Holt, R. A. Proposed experiment to test local hidden-variable theories. Phys. Rev. Lett. 23, 880 (1969).

5. Freedman, S. J. \& Clauser, J. F. Experimental test of local hidden-variable theories. Phys. Rev. Lett. 28, 938 (1972).

6. Aspect, A., Dalibard, J. \& Roger, G. Experimental test of Bell's inequalities using time-varying analyzers. Phys. Rev. Lett. 49, 1804 (1982).

7. Weihs, G., Jennewein, T., Simon, C., Weinfurter, H. \& Zeilinger, A. Violation of Bell's inequality under strict Einstein locality conditions. Phys. Rev. Lett. 81, 5039 (1998).

8. Rowe, M. A. et al. Experimental violation of a Bell's inequality with efficient detection. Nature 409, 791 (2001).

9. Matsukevich, D. N., Maunz, P., Moehring, D. L., Olmschenk, S. \& Monroe, C. Bell inequality violation with two remote atomic qubits. Phys. Rev. Lett. 100, 150404 (2008).

10. Ansmann, M. et al. Violation of Bell's inequality in Josephson phase qubits. Nature 461, 504 (2009).

11. Hofmann, J. et al. Heralded entanglement between widely separated atoms. Science 337, 72 (2012).

12. Giustina, M. et al. Bell violation using entangled photons without the fairsampling assumption. Nature 497, 227 (2013).

13. Christensen, B. G. et al. Detection-loophole-free test of quantum nonlocality, and applications. Phys. Rev. Lett. 111, 130406 (2013).

14. Hensen, B. et al. Loophole-free Bell inequality violation using electron spins separated by 1.3 kilometres. Nature 526, 682 (2015).

15. Giustina, M. et al. Significant-loophole-free test of Bell's theorem with entangled photons. Phys. Rev. Lett. 115, 250401 (2015).

16. Shalm, L. K. et al. Strong loophole-free test of local realism. Phys. Rev. Lett. 115, 250402 (2015).

17. Brunner, N., Cavalcanti, D., Pironio, S., Scarani, V. \& Wehner, S. Bell nonlocality. Rev. Mod. Phys. 86, 419 (2014).

18. Silva, R., Gisin, N., Guryanova, Y. \& Popescu, S. Multiple observers can share the nonlocality of half of an entangled pair by using optimal weak measurement. Phys. Rev. Lett. 114, 250401 (2015).

19. Pironio, S. et al. Random numbers certified by Bell's theorem. Nature 464, 1021 (2010).

20. Curchod, F. J. et al. Unbounded randomness certification using sequences of measurements. arXiv:1510.03394v1 [quant-ph].

21. Wiseman, H. W., Jones, S. J. \& Doherty, A. C. Steering, entanglement, nonlocality, and Einstein-Podolsky-Rosen Paradox. Phys. Rev. Lett. 98, 140402 (2007).

22. Händchen, V. et al. Observation of one-way Einstein-Podolsky-Rosen steering. Nat. Photon. 6, 596 (2012).

23. Aharonov, Y., Albert, D. Z. \& Vaidman, L. How the result of a measurement of a component of the spin of a spin-1/2 particle can turn out to be 100 . Phys. Rev. Lett. 60, 1351 (1988).

24. Hosten, O. \& Kwiat, P. Observation of the spin Hall effect of light via weak measurements. Science 319, 787 (2008)

25. Dixon, P. B., Starling, D. J., Jordan, A. N. \& Howell, J. C. Ultrasensitive beam deflection measurement via interferometric weak value amplification. Phys. Rev. Lett. 102, 173601 (2009).
26. $\mathrm{Xu}, \mathrm{X}$. Y. et al. Phase estimation with weak measurement using a white light source. Phys. Rev. Lett. 111, 033604 (2013).

27. Lundeen, J. S., Sutherland, B., Patel, A., Stewart, C. \& Bamber, C. Direct measurement of the quantum wavefunction. Nature 474, 188 (2011).

28. Lundeen, J. S. \& Bamber, C. Procedure for direct measurement of general quantum states using weak measurement. Phys. Rev. Lett. 108, 070402 (2012).

29. Aharonov, Y., Botero, A., Popescu, S., Reznik, B. \& Tollakson, J. Revisiting Hardy's paradox: counterfactual statements, real measurements, entanglement and weak values. Phys. Lett. A 301, 130 (2002).

30. von Neumann, J. Mathematical Foundations of Quantum Mechanics. ((Princeton University Press, Princeton, 1955).

31. Cirel'son, B. S. Quantum generalizations of Bell's inequality. Lett. Math. Phys. 4, 93 (1980).

32. Higgins, B. L., Palsson, M. S., Xiang, G. Y., Wiseman, H. W. \& Pryde, G. J. Using weak values to experimentally determine "negative probabilities" in a two-photon state with Bell correlations. Phys. Rev. A 91, 012113 (2015).

33. Mal, S., Majumdar, A. S. \& Home, D. Sharing of nonlocality of a single member of an entangled pair of qubits is not possible by more than two unbiased observers on the other wing. Mathematics 4, 48 (2016).

34. Kraus, K. States, Effects, and Operation. (Springer-Verlag, Berlin, 1983).

35. Fedrizzi, A., Herbst, T., Poppe, A., Jennewein, T. \& Zeilinger, A. A wavelengthtunable fiber-coupled source of narrowband entangled photons. Opt. Express 15 15377 (2007).

36. Li, Y., Zhou, Z. Y., Ding, D. S. \& Shi, B. S. CW-pumped telecom band polarization entangled photon pair generation in a Sagnac interferometer. Opt. Express 23 28792 (2015).

37. Gisin, N., Ribordy, G., Tittel, W. \& Zbinden, H. Quantum cryptography. Rev. Mod. Phys. 74, 145 (2002).

38. Scarani, V. et al. The security of practical quantum key distribution. Rev. Mod. Phys. 81, 1301 (2009).

39. Goldenberg, L., Vaidman, L. \& Wiesner, S. Quantum gambling. Phys. Rev. Lett. 82, 3356 (1999).

40. Zhang, P. et al. Optical realization of quantum gambling machine. EPL 82, 30002 (2008).

41. Georgescu, I. M., Ashhab, S. \& Nori, F. Quantum simulation. Phys. Rev. Mod. 86, 153 (2014).

42. Schiavon, M., Calderaro, L., Pitaluga, M., Vallone, G. \& Villoresi, P. Three-observer Bell inequality violation on a two-qubit entangled state. Quantum Sci. Technol. 2, 015010 (2017).

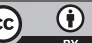

Open Access This article is licensed under a Creative Commons Attribution 4.0 International License, which permits use, sharing, adaptation, distribution and reproduction in any medium or format, as long as you give appropriate credit to the original author(s) and the source, provide a link to the Creative Commons license, and indicate if changes were made. The images or other third party material in this article are included in the article's Creative Commons license, unless indicated otherwise in a credit line to the material. If material is not included in the article's Creative Commons license and your intended use is not permitted by statutory regulation or exceeds the permitted use, you will need to obtain permission directly from the copyright holder. To view a copy of this license, visit http://creativecommons. org/licenses/by/4.0/.

(c) The Author(s) 2018 